\title{
Clinical and economic impact of the implementation of an antimicrobial stewardship program in four Colombian healthcare institutions
}

Christian Pallares ( $\square$ icako@hotmail.com )

Cristhian Hernández-Gómez

Universidad El Bosque

Sergio Reyes

Centro Internacional de Entrenamiento e Investigaciones Medicas

Kevin Escandón-Vargas

Centro Internacional de Entrenamiento e Investigaciones Medicas

Tobias Manuel Appel

Universidad El Bosque https://orcid.org/0000-0001-6413-5623

\section{Soraya Salcedo}

Clinical del Norte, Barranquilla

Lorena Matta

Corporación Clínica Universitaria Comfenalco

Ernesto Martínez

Hospital Universitario del Valle Evaristo García E.S.E.

\section{Sara Cobo}

DIME Clínica Neurovascular

\section{Laura Mora}

Clínica General del Norte

\section{Adriana Marín}

Corporacion de Estudios Tecnologicos del Norte del Valle

\section{María Virginia Villegas}

Universidad El Bosque

Research article

Keywords: Antimicrobial stewardship, Latin America, Colombia, healthcare economics

Posted Date: April 18th, 2019

DOI: https://doi.org/10.21203/rs.2.9215/v1 
License: (c) (i) This work is licensed under a Creative Commons Attribution 4.0 International License. Read Full License 


\section{Abstract}

Background Despite multiple reports of the benefits of antimicrobial stewardship programs (ASPs) in developed countries, regarding patient safety, decrease in antimicrobial resistance, consumption and spending on antimicrobials, there is very limited evidence of the same benefits in a different economic and social context. The objective of this study was to evaluate the clinical and economic impact of the implementation of an ASP in Colombia. Methods We conducted a multicenter, quasi-experimental cohort study in four Colombian healthcare institutions between 2007 and 2014 including 900 patients (429 patients before and 471 after the implementation of the ASP) to evaluate the impact of these programs. Results Compared to the pre-ASP, the post-ASP group showed a greater adherence to the use of the empirical therapy and de-escalation based on the guidelines (45\% vs. $9 \%$ and $92 \%$ vs. $8 \%$, respectively; $p<$ $0.001)$, as well as a higher proportion of clinical cure at the end of treatment and lower mortality ( $93 \% \mathrm{vs.}$ $74 \%$ and $14 \%$ vs. $28 \%$, respectively; $p<0.001$ ). In the post-ASP patient group the opportunity for clinical improvement was 10 times greater compared with the pre-ASP group (adjusted OR $=10.4095 \% \mathrm{Cl} 1.21$ 89.41 ) and there were less complications following targeted management (adjusted OR $=0.4995 \% \mathrm{Cl}$ 0.25-0.97). Furthermore, after the implementation of the ASP there was lower average expenditure associated with hospital stay, laboratory tests, infectious diseases consultation, and empirical antimicrobial therapy choice. Conclusion With this study, we were able to show that even in limited income countries, ASPs are feasible to implement.

\section{Background}

A growing body of evidence demonstrates that strategies dedicated to promote the rational and effective use of antimicrobial agents including antimicrobial selection, dosage, route of administration and duration of therapy, commonly referred to as antimicrobial stewardship programs (ASPs), are a key component to address antimicrobial resistance in hospital and ambulatory healthcare settings and even in agriculture $(1,2)$. Besides, ASPs have been shown to improve patient care by enhancing optimal therapy with lower rates of treatment failure and reducing collateral damage associated with antimicrobial administration, intravenous lines and length of hospital stay. Moreover, these programs often achieve these benefits while saving hospitals money (3).

In Latin America, many countries are in the process of developing ASPs. However, although the effectiveness of these strategies is proven in North-American and European countries to have a positive impact on patient care, antimicrobial resistance and healthcare economics $(4,5)$, there is very few literature on the impact of ASPs in developing countries in general and even less in Latin American countries (6). In addition, these studies mainly focus on the impact of ASPs on antimicrobial consumption and resistance. This situation is concerning since healthcare systems in developing countries count with very limited resources, meanwhile antimicrobial resistance rates usually are higher compared to developed countries (6-8). 
Therefore, the objective of the current study was to determine the clinical and economic impact of the implementation of an ASP in four Colombian healthcare institutions in patients with community-and healthcare-acquired infections.

\section{Methods}

A multicenter, quasi-experimental, retrospective cohort study was conducted between January 2007 and December 2014 comparing clinical and economic outcomes in patients with community-acquired infections (CAls) and healthcare-acquired infections (HAls) before and after the implementation of an ASP (Figure 1).

\section{Implementation and structure of the ASP}

The implementation process took between 6 and 12 months and was executed individually in each of the four institutions. All hospital ASPs had several characteristics in common: (A) the multidisciplinary antimicrobial stewardship (AMS) team was led by an infectious disease specialist; (B) all participating institutions had previously established antimicrobial guidelines; (C) infectious disease specialists, epidemiologists, pharmacists, microbiologists and general practitioners were part of the multidisciplinary team; (D) new guidelines for the most prevalent infectious diseases were elaborated based on the local microbiology or existing guidelines were updated by the ASP team and specialists of different clinical areas; $(E)$ antimicrobial guidelines were socialized with all healthcare professionals; $(F)$ active antimicrobial prescription surveillance with constant feedback regarding appropriateness (according to institutional guidelines) towards prescribers was performed by a general practitioner in three out of the four hospitals, in contrast with only one institution that implemented restrictive prescription control; $(\mathrm{G})$ educational activities were implemented in every participating institution according to their needs.

\section{Study population}

We included patients aged 18 years and older with CAls and HAls, from four tertiary care institutions in two Colombian cities (Cali and Barranquilla) (Table 1). We excluded patients receiving only peri-operative surgical prophylaxis, those with fungal infections, those whose care was limited to the emergency department, those with infections not included in the institutional antimicrobial guidelines, and those with incomplete clinical records. Inclusion and exclusion criteria were applied retrospectively.

Sample size was estimated for the outcome of "adherence to antimicrobial guidelines" based on a confidence level of $95 \%$ and statistical power of $80 \%$, assuming an adherence of $70-90 \%$ among participating institutions and a $10 \%$ adjustment for non-response.

\section{Clinical outcomes}

We collected information that included sociodemographic data, patient location in the hospital, diagnosis of infection, comorbidities, antimicrobial therapy during patient care, and complications during the hospital stay. Treatment prescribed during the first 24 hours of hospitalization was considered to be the 
initial treatment and was recorded whether or not adhering to guidelines. Regarding clinical outcomes definitions, adequate empirical dose was defined as the administration of the antimicrobial dose established by the institutional guidelines; adherence to antimicrobial guidelines was defined as the accordance between the antibiotics chosen by the attending physician (for the diagnosed infection) and the recommendations included in the institutional guidelines (9); adequate de-escalation was defined as adjusting initial, adequate broad-spectrum treatment by changing the antimicrobial agent or discontinuing an antimicrobial combination according to the patient's culture results (10). Mortality was defined as death in the first 30 days following hospital admission.

The primary outcome was clinical improvement at 72 hours of empirical treatment, as well as clinical improvement at 72 hours of targeted treatment. Clinical improvement was defined as the resolution of the systemic inflammatory response after three days of empirical treatment or guided by antibiogram. Clinical cure at the end of treatment was defined as the resolution of all the signs and symptoms of the infection at the end of antibiotic treatment. For systemic inflammatory response syndrome (SIRS), sepsis and septic shock, the definitions of the 2001 SCMM/ESCIM/ACCP/ATS/SIS International Sepsis Definitions Conference were applied (11).

\section{Economic measures}

The cost of care for patients with infections that were selected for the study corresponded to the sum of the infectious diseases consultations, antibiotic consumption, clinical laboratory tests, microbiological cultures and the length of hospital stay per patient. A partial economic assessment was carried out using micro-costing techniques as a way of determining the magnitude of the resources spent. The allocation of costs for each resource is based on a reference cost and is adjusted by inflation rate based on the Colombian manual document for costs (SOAT 2017 Tariff Manual).

\section{Statistical analyses}

Chi-square test or Fisher exact test were used to compare proportions between the two groups. Nonparametric statistics were calculated depending on the normality of the distribution. For the bivariate analysis, the odds ratio (OR) with $95 \%$ confidence interval $(95 \% \mathrm{Cl})$ was established independently for each variable of interest. In the multivariable analysis, logistic regression was carried out with the variables that presented a $p$ value $<0.20$ in the bivariate analysis. All $p$ values $<0.05$ were considered statistically significant.

This research was classified as minimal risk and the study was approved by the ethics committees of the participating institutions and the Centro Internacional de Entrenamiento e Investigaciones Médicas (CIDEIM) ethics and research committee.

\section{Results}


We included 900 patients with bacterial infections who were hospitalized in the participating institutions, including 471 exposed patients (post-implementation of the ASP) and 429 non-exposed patients (preimplementation of the ASP) (Table 2).

\section{Clinical outcomes of the ASP}

The median age of included patients was 62 years and $54 \%$ of the patients were males. Before hospital admission, $52 \%$ were admitted from the community and $48 \%$ from other hospital or healthcare institutions; $70 \%$ of the patients had at least one comorbidity. Urinary tract infections were the most common infections among all patients $(28 \%)$, followed by bloodstream infections $(26 \%)$.

Statistically significant differences were present between pre- and post-ASP groups regarding comorbidities, previous history of surgery after admission, sepsis and septic shock at the time of infection (Table 2 and Table 3). Compared to the pre-ASP, the post-ASP group showed a greater adherence to the use of the empirical therapy and de-escalation based on the guidelines ( $45 \%$ vs. $9 \%$ and $92 \%$ vs. $8 \%$, respectively; $p<0.001$ ), as well as a higher proportion of clinical cure at the end of treatment and lower mortality ( $93 \%$ vs. $74 \%$ and $14 \%$ vs. $28 \%$, respectively; $p<0.001$ ). In the post-ASP patient group, the opportunity for clinical improvement was 10 times greater compared with the pre-ASP group (adjusted $\mathrm{OR}=10.4095 \% \mathrm{Cl} 1.21-89.41$ ) and there were less complications following targeted management (adjusted OR $=0.49$ 95\% Cl 0.25-0.97) (Table 4 and Table 5).

\section{Economic outcomes for the ASP}

The expenditure on healthcare for infected patients not included in the ASP was higher compared to the patients who were included in the ASP $(4,156$ USD vs 2,952 USD, $p<0.001)$ (Table 6). When each item was individually analyzed and entered in the statistical analysis, there was significantly higher average expenditure related to hospital stay, laboratory tests, infectious diseases consultation, and empirical antimicrobial therapy choice in the patients not included in the ASP $(p<0.001)$. On the other hand, there was no statistically significant difference with regard to the targeted antimicrobial therapy choice. The monthly average cost for each of the ASPs was 3,785 USD (ranging between 2,469 and 5,488 USD).

\section{Discussion}

Despite the well acceptance of ASPs as a routine measure of quality improvement, these programs consist of a bundle of interventions, making it difficult to assess the impact of a single intervention and therefore a clear consensus is lacking (12). Outcome assessment in the pre- and post-ASP implementation periods becomes the most commonly employed study design, despite all its limitations, to mention, the retrospective nature and the quasi-experimental design of the study (13).

We consider that there are several elements that might have contributed to the observed outcomes. First, all four hospitals had antimicrobial guidelines based on their epidemiology elaborated by an infectious disease physician in conjunction with other relevant specialists. Inclusion of a multidisciplinary group to 
approve and adopt the antimicrobial guidelines likely increased its adherence, as it is suggested by other studies (14). Second, all hospitals had a steward dedicated to the ASP at least part time. This person was previously trained in AMS concepts and strategies, hospital epidemiology, mechanisms of resistance in gram-negative and gram-positive bacteria present in each hospital, and when de-escalation was an option. Third, the steward was supported by the on-service infectious disease physician, the infection prevention and control committee, and the general manager of the hospital for any discrepancies in the therapeutic decision-making between the steward and the primary doctor in charge of the patient. These discussions frequently favored the steward recommendation to change the antibiotic (empirical, therapeutic or de-escalation). The adherence to de-escalation in the post-ASP period was greater (92\%) compared to the pre-ASP period, which was only $8 \%$. This suggests a very high credibility achieved by the ASP. Fourth, all but one hospital implemented prospective audit and feedback which, although is more time-consuming compared to the antibiotic pre-authorization strategy, generally is more accepted, resulting in higher clinical benefits such as less complications after the empirical antibiotic was started and greater proportion of adequate dosing (15).

Patients under the ASP in our study had a $14 \%$ reduction in mortality compared with patients prior to ASP implementation. A significant clinical improvement was achieved after ASP implementation, even though in the post-ASP period patients had statistically significant more neurological comorbidities, and an increased likelihood of sepsis at the moment of infection. There were no differences between the pre- and post-ASP groups regarding age and type of infection. The other benefit shown in the post-ASP was length of stay, which decreased 4 days in average. Our results are similar to a publication from 2013, which demonstrated that non-adherence to hospital antibiotic guidelines in a public hospital in Colombia, was an independent risk factor for mortality from HAls (16).

Finally, our study suggests an economic benefit associated with the ASP in each hospital. The average expenditure associated with hospital stay, laboratory tests, infectious diseases consultation, and empirical antimicrobial therapy choice was higher for patients in the pre-ASP period. Our study did not evaluate other costs not directly related to infections. Nevertheless, some studies suggest that ASPs are able to reduce non-infectious disease expenditures related with adverse events or complications during hospital stay (17).

Our results are in line with what was previously described by Dik et al (1), who showed that ASPs in a short term are able to improve patient care by increasing adherence to optimal antimicrobial therapy and, in a short to intermediate term, reduce collateral damage. These effects are due to a lower exposure to antimicrobial toxicity, fewer Clostridioides difficile infections, reduced number of catheter-associated infections due to shorter intravenous treatment regimens, and less non-infectious complications associated with shorter hospital stay as described previously. In an intermediate to long term, ASP benefits are usually the reduction of antimicrobial prescription as well as bacterial resistance. All previously described factors contribute to a reduction of hospital costs.

\section{Conclusions}


This study comparing a pre- versus a post-ASP implementation period in four Colombian hospitals was able to show the feasibility of implementing ASPs along with optimization of healthcare expenditures, confirming that ASPs may have a great impact for Latin American countries and other resource-limited countries.

\section{List Of Abbreviations}

AMS: Antimicrobial Stewardship

ASP: Antimicrobial Stewardship Program

aOR: Adjusted odds ratio

CAI: Community-acquired infection

Cl: Confidence interval

HAl: Healthcare-acquired infection

NS: Not significant

OR: Odds ratio

SD: Standard deviation

SIRS: Systemic inflammatory response syndrome USD: US dollars

\section{Declarations}

\section{Ethical approval and consent to participate}

This research was classified as minimal risk and the study was approved by the ethics committees of the participating institutions and the Centro Internacional de Entrenamiento en Investigaciones Médicas (CIDEIM) ethics and research committee. Patient data was obtained retrospectively with permission of the participating institutions after approval by the ethics committees. According to the national legislation, consent to participate was not required.

Consent for publication

All authors agreed on the publication of the research.

Availability of supporting data

Not applicable 


\section{Competing interests}

Maria V. Villegas has received consulting fees and research grants from Merck Sharp \& Dohme, Pfizer, Merck SA, West SA and OpGen. Ernesto Martínez hast received consulting fees from Pfizer, Merck Sharp and Dohme, Stendhal, Gilead/Gador, GSK and ABBVIE. Christian Pallares has received consulting fees and research grants from Merck Sharp \& Dohme, Pfizer, Merck SA, West SA and OpGen. Cristhian Hernández-Gómez has received consulting fees from Merck Sharp \& Dohme, Pfizer and West. Cristhian Hernández-Gómez participated in research activities until august 2018.

\section{Funding}

None

\section{Author's contributions}

Cristhian Hernández-Gómez and Christian Pallares designed and implemented the study. Kevin Escandón-Vargas, Sergio Reyes, Soraya Salcedo, Lorena Matta, Ernesto Martínez, Sara Cobo, Laura Mora, Adriana Marín, Adriana Correa sopported the implementation and discussed and analyed the results. Cristhian Hernández-Gómez, Christian Pallares, Tobias Manuel Appel and María Virginia Villegas discussed and analyzed the results and wrote the paper.

Acknowledgements

We want to thank the professionals assigned to the services of microbiology, pharmacy, infection control, hospital epidemiology and infectious diseases of the institutions participating in the study.

\section{References}

1. Dik J-WH, Hendrix R, Poelman R, Niesters HG, Postma MJ, Sinha B, et al. Measuring the impact of antimicrobial stewardship programs. Expert Rev Anti Infect Ther [Internet]. 2016 Jun 2 [cited 2019 Feb 2];14(6):569-75. Available from: http://www.ncbi.nlm.nih.gov/pubmed/27077229

2. Davey P, Marwick CA, Scott CL, Charani E, McNeil K, Brown E, et al. Interventions to improve antibiotic prescribing practices for hospital inpatients. Cochrane Database Syst Rev [Internet]. 2017 Feb 9 [cited 2018 Nov 27];2:CD003543. Available from: http://www.ncbi.nlm.nih.gov/pubmed/28178770

3. Laxminarayan R, Duse A, Wattal C, Zaidi AKM, Wertheim HFL, Sumpradit N, et al. Antibiotic resistancethe need for global solutions. Lancet Infect Dis [Internet]. 2013 Dec 1 [cited 2018 Nov 27];13(12):105798. Available from: http://www.ncbi.nlm.nih.gov/pubmed/24252483

4. Ruttimann S, Keck B, Hartmeier C, Maetzel A, Bucher HC. Long-Term Antibiotic Cost Savings from a Comprehensive Intervention Program in a Medical Department of a University-Affiliated Teaching Hospital. Clin Infect Dis [Internet]. 2004 Feb 1 [cited 2018 Sep 17];38(3):348-56. Available from: http://www.ncbi.nlm.nih.gov/pubmed/14727204 
5. Roberts RR, Hota B, Ahmad I, Scott II RD, Foster SD, Abbasi F, et al. Hospital and Societal Costs of Antimicrobial-Resistant Infections in a Chicago Teaching Hospital: Implications for Antibiotic Stewardship. Clin Infect Dis [Internet]. 2009 Oct 15 [cited 2018 Nov 27];49(8):1175-84. Available from: http://www.ncbi.nlm.nih.gov/pubmed/19739972

6. Aryee A, Price N. Antimicrobial stewardship - can we afford to do without it? Br J Clin Pharmacol [Internet]. 2015 Feb [cited 2019 Feb 4];79(2):173-81. Available from:

http://www.ncbi.nlm.nih.gov/pubmed/24803175

7. Shears P. Epidemiology and surveillance of antimicrobial resistance in the tropics. Trans R Soc Trop Med Hyg [Internet]. 2001 Mar 1 [cited 2019 Feb 4];95(2):127-30. Available from: https://www.sciencedirect.com/science/article/pii/S0035920301901348

8. Ayukekbong JA, Ntemgwa M, Atabe AN. The threat of antimicrobial resistance in developing countries: causes and control strategies. Antimicrob Resist Infect Control [Internet]. 2017 Dec 15 [cited 2019 Feb 4];6(1):47. Available from: http://aricjournal.biomedcentral.com/articles/10.1186/s13756-017-0208-x

9. van der Velden LBJ, Tromp M, Bleeker-Rovers CP, Hulscher M, Kullberg BJ, Mouton JW, et al. Nonadherence to antimicrobial treatment guidelines results in more broad-spectrum but not more appropriate therapy. Eur J Clin Microbiol Infect Dis [Internet]. 2012 Jul [cited 2019 Feb 4];31(7):1561-8. Available from: http://www.ncbi.nlm.nih.gov/pubmed/22108843

10. Ohji G, Doi A, Yamamoto S, Iwata K. Is de-escalation of antimicrobials effective? A systematic review and meta-analysis. Int J Infect Dis [Internet]. 2016 Aug 1 [cited 2019 Feb 4];49:71-9. Available from: https://www.sciencedirect.com/science/article/pii/S1201971216310852

11. Levy MM, Fink MP, Marshall JC, Abraham E, Angus D, Cook D, et al. 2001 SCCM/ESICM/ACCP/ATS/SIS International Sepsis Definitions Conference. Crit Care Med [Internet]. 2003 Apr [cited 2019 Feb 1];31(4):1250-6. Available from: http://www.ncbi.nlm.nih.gov/pubmed/12682500

12. Davey P, Brown E, Charani E, Fenelon L, Gould IM, Holmes A, et al. Interventions to improve antibiotic prescribing practices for hospital inpatients. In: Davey P, editor. Cochrane Database of Systematic Reviews [Internet]. Chichester, UK: John Wiley \& Sons, Ltd; 2013 [cited 2019 Feb 4]. p. CD003543. Available from: http://www.ncbi.nlm.nih.gov/pubmed/23633313

13. Harris AD, McGregor JC, Perencevich EN, Furuno JP, Zhu J, Peterson DE, et al. The use and interpretation of quasi-experimental studies in medical informatics. J Am Med Inform Assoc [Internet]. 2006 [cited 2019 Feb 4];13(1):16-23. Available from: http://www.ncbi.nlm.nih.gov/pubmed/16221933

14. Shanbhag D, Graham ID, Harlos K, Haynes RB, Gabizon I, Connolly SJ, et al. Effectiveness of implementation interventions in improving physician adherence to guideline recommendations in heart failure: a systematic review. BMJ Open [Internet]. 2018 Mar 6 [cited 2019 Feb 6];8(3):e017765. Available from: http://www.ncbi.nlm.nih.gov/pubmed/29511005 
15. Dellit TH, Owens RC, McGowan JE, Gerding DN, Weinstein RA, Burke JP, et al. Infectious Diseases Society of America and the Society for Healthcare Epidemiology of America Guidelines for Developing an Institutional Program to Enhance Antimicrobial Stewardship. Clin Infect Dis [Internet]. 2007 Jan 15 [cited 2019 Feb 4];44(2):159-77. Available from: http://www.ncbi.nlm.nih.gov/pubmed/17173212

16. Pallares CJ, Martínez E, Martínez E. Factores de riesgo asociados a mortalidad en infecciones relacionadas con la atención en salud en un hospital universitario de tercer nivel en Colombia. Biomédica [Internet]. 2013 Nov 29 [cited 2019 Feb 4];34(0):148. Available from:

http://www.revistabiomedica.org/index.php/biomedica/article/view/1646

17. Ingeman A, Andersen G, Hundborg HH, Svendsen ML, Johnsen SP. In-Hospital Medical Complications, Length of Stay, and Mortality Among Stroke Unit Patients. Stroke [Internet]. 2011 Nov [cited 2019 Feb 4];42(11):3214-8. Available from: http://www.ncbi.nlm.nih.gov/pubmed/21868737

\section{Tables}

Table 1. Characteristics of involved institutions.

\begin{tabular}{lllll} 
Institution* & A & B & C & D \\
\hline City & Barranquilla & Cali & Cali & Cali \\
\hline Number of beds & 500 & 900 & 120 & 150 \\
\hline Type of hospital & Private & Public & Private & Private \\
\hline Clinical area & $\begin{array}{l}\text { General } \\
\text { hospital }\end{array}$ & $\begin{array}{l}\text { General } \\
\text { hospital }\end{array}$ & $\begin{array}{l}\text { General } \\
\text { hospital }\end{array}$ & $\begin{array}{l}\text { Cardiovascular and } \\
\text { neurosurgery }\end{array}$ \\
\hline $\begin{array}{l}\text { Organ } \\
\text { transplantation }\end{array}$ & No & Bone marrow & Bone marrow & No
\end{tabular}

*All general hospitals had emergency departments, general wards, intensive care units and general and specialized surgery.

Table 2. Distribution of baseline characteristics of patients exposed (Post-) and not exposed (Pre-) to the ASP. 


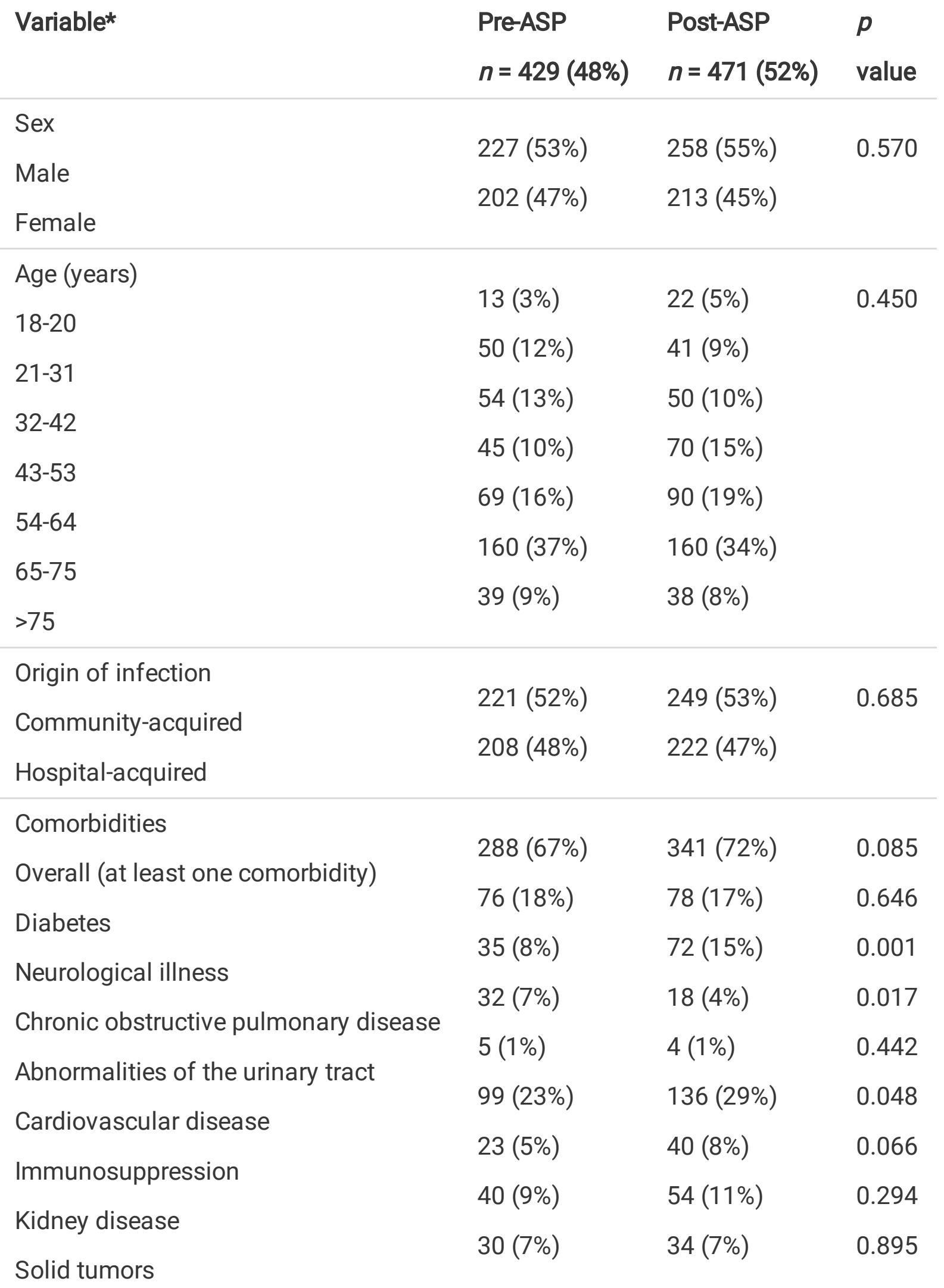

Abbreviation: ASP, antimicrobial stewardship program.

*Data are expressed as number (\%) unless specified otherwise.

Table 3. Distribution of clinical variables of patients exposed (Post-) and not exposed (Pre-) to the ASP. 
$n=429$

(48\%)

SIRS at initiation of treatment

Sepsis at initiation of treatment

Septic shock at initiation of treatment

Length of stay before infection, days, mean (SD) (HAI patients only)

Type of infection

Urinary tract infection

Bloodstream infection

Intra-abdominal infection

Pneumonia

Skin and soft tissue infection

Hospital location when the infection was diagnosed

Emergency department

Hospitalization

Dialysis and chemotherapy units

Intermediate care unit

Intensive care unit

Length of stay during infection, days, mean (SD)

Time to initiation of empirical antibiotic therapy, hours, mean (SD)

Adequate dose of empirical treatment

Adherence to empirical antibiotic therapy guidelines

Post-empirical treatment complications

Change in empirical treatment

Adequate de-escalation

Post-targeted treatment complications

Clinical improvement at 72 hours of empirical treatment

Clinical improvement at 72 hours of targeted treatment
$269(63 \%)$

$228(53 \%)$

$41(10 \%)$

$19.5(+/-$

24)

107 (25\%) 144 (31\%)

0.112

$106(25 \%) \quad 131$ (28\%)

$51(12 \%) \quad 50(11 \%)$

$92(21 \%)$

$73(17 \%)$

$27(6 \%)$

$11(2 \%)$

0.001

$175(41 \%) \quad 220(47 \%)$

$7(2 \%)$

$22(5 \%)$

$23(5 \%)$

$28(6 \%)$

$197(46 \%)$

$14.0(+/-8)$

$10.8(+/-7)$

$<0.001$

$5(+/-5)$

$4(+/-4)$

0.037

$33(25 \%)$

$96(86 \%)$

$<0.001$

$31(9 \%)$

$173(45 \%)$

$<0.001$

$185(43 \%)$

$166(35 \%)$

0.015

$190(52 \%) \quad 263(67 \%) \quad<0.001$

$23(8 \%)$

$311(92 \%)$

$<0.001$

$79(18 \%)$

$82(17 \%)$

0.694

$227(62 \%) \quad 285(73 \%)$

0.299

$160(73 \%) \quad 229(75 \%)$

0.663 
Clinical cure at the end of treatment

Mortality
$314(74 \%)$

$117(28 \%)$
$438(93 \%)$

$65(14 \%)$
$<0.001$

$<0.001$

Abbreviations: ASP, antimicrobial stewardship program; SD, standard deviation; SIRS, Systemic inflammatory response syndrome.

*Data are expressed as number (\%) unless specified otherwise.

Table 4. Bivariate analysis of patients exposed (Post-) and not exposed (Pre-) to the ASP.

\section{Variable}

Exposure to an ASP program

Sex

Age

Origin of infection (community- versus hospital-acquired)

Comorbidities

Sepsis

Septic shock

Time to initiate empirical antibiotic

Change in empirical treatment

Adherence to empirical antibiotic therapy guidelines

Adequate dose of empirical treatment

Post-empirical treatment complications

Post-targeted treatment complications

Septic shock after targeted therapy

Adequate de-escalation
OR (95\% Cl / p value)

$4.90(3.16-7.76 / p<0.01)$

$0.97(0.66-1.41 / p=0.879)$

$1.05(0.56-1.86 / p=0.846)$

$0.65(0.44-0.95 / p=0.021)$

$1.22(0.81-1.83 / p=0.290)$

$1.48(1.01-2.16 / p=0.033)$

$0.20(0.11-0.36 / p<0.001)$

$1.23(0.63-2.41 / p=0.504)$

$1.33(0.89-1.98 / p=0.138)$

$3.35(1.88-6.34 / p<0.001)$

$1.48(0.68-3.25 / p=0.272)$

$0.38(0.25-0.59 / p<0.001)$

$1.10(0.96-1.28 / p=0.062)$

$0.23(0.14-0.38 / p<0.001)$

$3.76(2.21-6.60 / p<0.001)$

Abbreviation: ASP, antimicrobial stewardship program; $\mathrm{Cl}$, confidence interval; OR, odds ratio.

Table 5. Logistic regression model in patients exposed (Post-) and not exposed (Pre-) to the ASP. 
Variable

Exposure to an ASP program

Origin of infection (community-versus hospital-acquired)

Sepsis

Septic shock

Post-empirical treatment complications

Post-targeted treatment complications

Change in empirical treatment

Adherence to empirical antibiotic therapy guidelines

Sepsis after targeted therapy

Septic shock after targeted therapy

Adequate de-escalation
aOR (95\% Cl / p value)

$10.40(1.21-89.41 / p=0.033)$

$0.77(0.44-1.37 / p=0.386)$

$1.57(0.82-3.01 / p=0.170)$

$0.81(0.31-2.09 / p=0.669)$

$0.68(0.27-1.72 / p=0.428)$

$0.49(0.25-0.97 / p=0.041)$

$1.21(0.57-2.59 / p=0.611)$

$1.10(0.49-2.47 / p=0.813)$

$0.64(0.25-1.64 / p=0.362)$

$0.37(0.13-1.07 / p=0.068)$

$0.52(0.64-4.28 / p=0.550)$

Abbreviation: aOR, adjusted odds ratio; ASP, antimicrobial stewardship program; $\mathrm{Cl}$, confidence interval.

Table 6. Cost analysis of patients exposed (Post-) and not exposed (Pre-) to the ASP.

\begin{tabular}{llll} 
Variable & Pre-ASP & Post-ASP & p value \\
& $\boldsymbol{n}=\mathbf{4 2 9}(\mathbf{4 8 \%})$ & $\boldsymbol{n}=\mathbf{4 7 1}(\mathbf{5 2 \%})$ & \\
\hline Cost of stay during infection (USD) * & 3,669 & 2,436 & $<0.001$ \\
\hline Cost of exams and initial intervention (USD) * & 275 & 181 & $<0.001$ \\
\hline Cost of empirical therapy (USD) * & 363 & 208 & $<0.001$ \\
\hline Targeted therapy cost (USD) * & 71 & 57 & NS \\
\hline Total infection cost (USD) * & 4,156 & 2,952 & $<0.001$
\end{tabular}

Abbreviation: ASP, antimicrobial stewardship program; NS, not significant.

*Average cost per patient

\section{Figures}


A multicenter, quasi-experimental, retrospective cohort study in four Colombian hospitals

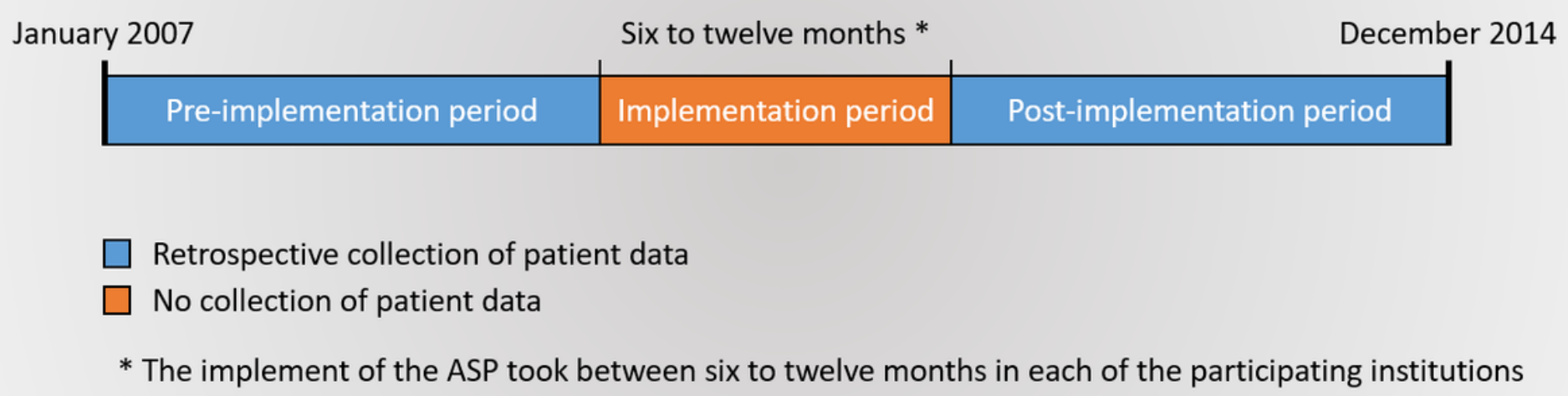

* The implement of the ASP took between six to twelve months in each of the participating institutions

\section{Figure 1}

Study design 